\title{
Symbolic Expression and Application in Graphic Design
}

\author{
Lanzhen Wang \\ Huanghe Science and Technology College \\ Zhengzhou, China 450000
}

\begin{abstract}
The essential relationship in graphic design and semiotics has been analyzed in this paper and the role of symbols in communication between human and culture as well as information has been discussed in combination with various examples in daily life such as image symbol, indicator and symbolic sign. Then the significance of recognizing and stressing symbols and their expressions have been analyzed further to strive to find symbols' feature and apply symbols in design more accurately. Finally, variable control has been proposed in choosing graphic design symbols, to guarantee designer's design ideas and inner feeling can be appropriately expressed in the works.
\end{abstract}

Keywords-symbol; semiotics; visualization; expressive thinking; graphic design

\section{INTRODUCTION}

There are many such examples in daily life, and similar applications also appear in the actual design, why? These phenomenon seems to be governed by some law? In fact, many techniques applied in graphic design are derived from semiotic principles, and these phenomena also appear in graphic design. In visual space design, graphic image itself is a symbol, and it is used as visual communication media with more direct and accurate characteristics. It plays an essential role in cultural exchange and information communication between people. Graphic design has been influenced by semiotics in terms of form expression thinking, meanwhile, the existence of semiotics has also enriched the expression skills of graphic design, and made the conveying of information more scientifically and accurately.

\section{ESSENTIAL RELATIONS BETWEEN GRAPHIC DESIGN AND SEMIOTICS}

\section{A. About Symbols and Semiotics}

Dating back to the Warring States period, a famous thinker appeared in China, namely Chuang Tzu. He mentioned in "Zhuangzi Outer Chapters" that the relationship between representation and the represented is the relationship between language and objects, which is taken as the media for information conveying. Language is used to convey information of objects, so it is the representation of objects, and objects will naturally become the represented by language. With reference to specific media, symbol has become a representative of certain objects or the substitute of this object. The view of Eco, a famous Italian expert in symbol, is that symbol can represent anything in the established social habits. Although the object $\mathrm{Y}$ is not here, but we can use $\mathrm{X}$ as a symbol to represent it. Just as people will think of Swan Goose when they see the quagmire and footprints on snow, and the number and size of Swan Goose can be learned through these footprints. Semiotics is also a science with its own sign laws.

\section{B. Origin of Symbols}

Unlike other creatures, human have their unique language and social activity, so the level of their conscious activities has been highly developed. Take the understanding representation as a starting point and sum up the experience of real life, people's thinking has gradually formed the concept of brain, and then form the image corresponding to the outside world and their own mind. Slowly, these images directly break away from the open external relations, and operate independently in mind. Based on the language in narrow sense, these images can appear in the form of visual graphics, body movements and music.

\section{Graphic Design and Principles of Semiotics}

The world has been symbolized in human consciousness, and our thinking is nothing but selection, combination, transformation and regeneration of symbols. So we can believe that the main body of thinking is symbol which is used by human as a tool for thinking. In the graphic design process, we need to plan the location, proportion and mutual relations of words in two-dimensional space in order to complete the transformation of information, this is without doubt a thinking process. And this way of thinking has extended from the designers to viewer. As the main body of thinking, symbol is the effective guarantee of this continuation. Go back to the above cases, danger and vigilance are represented by black and yellow stripes, which are the color of bee and tiger, conveying information in a driver's consciousness.

As part of the language in broad sense, graphic design relies on print media, with its essential purpose as conveying information in this way. Therefore, graphic design is consistent with the expression way of symbols very well, and the means it relies on are the symbolic methods and principles. Through the above analysis, it can be concluded that if we want to accomplish the purpose of information 
conveying, we must use symbols reasonably and accurately. The key of a success graphic design work, is to convey information of $\mathrm{Y}$ accurately using symbol X.

\section{EXPRESSION AND APPLICATION OF SYMBOLS}

Graphic design itself is a symbol in terms of expression methods. Designer' $\mathrm{s}$ thinking process and conclusions are conveyed to the audience through symbols with aims to guide and persuade audiences. In other words, the audience need to validate their own experience and feel the thoughts and feelings intended to be expressed by the designer by virtue of the designer's work. We can clearly see that, graphic design works have become a symbol of designer' $\mathrm{s}$ thoughts and feelings at this time, and link up designers' thoughts and feelings with that of audiences as a media. At this time, the success of designer's work is determined by whether or not the audiences can receive the message conveyed accurately, quickly and effectively. It can be seen the importance of selection, combination, transformation and regeneration of graphical symbols for designers' thinking process. In summary, the expression of thoughts and feelings can be made by virtue of symbols. Therefore, if we want to achieve the purpose of expressing thoughts and feelings, we must have a more in-depth understanding about the tool of symbol.

\section{A. Deepen Symbols Constantly}

Based on different referent objects, symbols can be divided into three different types with three different levels:

The first is image symbol, and the identification method of it is relatively straightforward, such as portrait takes a man as the symbol formed by mock objects.

The second is indicator which links symbol and referent object through causal or space-time relationship. For example, the indicator of a building' $\mathrm{s}$ exit is the gate.

The third is symbolic sign. Due to social customs or people' s longstanding imagination, the symbolic sign is agreed to be granted to referent object, for example, the revolutionary symbol is red.

These symbols are independent without influencing each other in terms of types, and show a gradual deepening state in terms of level. The information contents of symbols have been increasingly widened from image symbol, to indicator and then the symbolic sign. Here takes pigeon as an example. If a pigeon appears on food packaging, then it indicates the food inside has a relationship with pigeons, at this time it is the image symbol; pigeons are animals in our mind with the characteristics of familiar animals in our daily life, so when a pigeon appears on an indicator board, we will obtain such indicatory information of "Here is the field for animal conservation", then it becomes the indicator; we clearly know that pigeon is a symbol of peace, and when it appears together with the maple leaves, it represents a Canadian charity institute, so at this time, it is the symbolic sign. Obviously these three types of symbols do not interfere with each other, and the way information is communicated is also very effective. Its conversion in terms of level is inevitable, because there exists the inherent causality. Simple image symbol can become indicator, and people' s longstanding experience to it has given birth of their imagination agreeing to give it a more profound meaning, so it can be converted into symbolic sign. Thus, it can be seen that only through a targeted process to deepen the symbols, can the thoughts and feelings be conveyed effectively and accurately.

\section{B. Grasp Variable Thinking of Symbols}

Personal thinking is narrow, and the thinking activities of human society is broad. The former is equal to the life in terms of time, but the latter is eternal. The former is exploratory as for different living space, but the latter has adaptability. Symbol is the main body of thinking, which will change with the change of time and space. Specific symbol referent will be different along with different time and space. One same object can be represented by different symbols at different times and spaces. The grasp of these variables in design is a guarantee for symbol to convey information accurately.

Referent of specific symbols and temporal and spatial variation: Take Oracle as an example. It is the earliest evidence-based text found to record divination results and other records of the ancients. As a symbol, it has characteristics of recording events. And at present, thousands of years later, when the Oracle appears in graphic design works, although it is still a symbol, but the meaning of event recording has lost. It represents a symbolic sign with historical significance, conveying our memorial and glory.

In ancient times, "与斤" is a symbol of the fire or the sun, which is a symbol, talisman or religious symbol. "压" has appeared in many places, especially in China. It represents the Buddhist, and in Sanskrit it means "collection of auspiciousness". In 693 AD, Wu Zetian defined it shall be read as "wan". However, in modern Europe, this character represents a completely different meaning. In 1920, Hitler, the leader of German Nazi party defined it as a symbol of "the mission to strive for victory of Aryan". This indicates that the same symbol will generate completely different meaning in different historical spaces. The meaning of

“厉" popular in China and other Asian countries is not recognized by western countries, because they simply can not understand this symbolic meaning which different from their habits.

Social factors are also a key element affecting the symbolic significance. For example, the condom was just a simple birth control tool. But in recent decades, the referent of it as a symbol has changed a lot. The birth of it is only as far as its role is concerned. But with the spread of "AIDS", it frequently appears in "AIDS prevention" propaganda works, and people have begun to slowly come to accept its new image. And the popular network at present also have a similar experience. For example, the symbol "@" only represented the computer previously, but now it is often used in the theme graphic design.

Referent of the same object in different times and spaces: With the passage of time, the development of society can be 
described as fast-changing. And it is precisely the constant conversion of times and spaces that render objects the changing symbols and their meanings. Time and space are changing, and people's awareness of the same object is also constantly changing, so the image stored in t people' s brain, namely the symbols, will appear a variety of differences. The event that changes the fastest in society can be described as fashion, and symbols that represent fashion will naturally not stand still.

In 2002, "Mashimaro" set off a burst of fashion style. People who had seen this animated movie would greet each other with "Have you seen Mashimaro today?" At that time, it was a symbol representing "fashion". Many businesses were inspired and created some models that consistent with the hero' $s$ image in this movie, so that consumers would buy these things. And wasn' t such business strategy has exactly grasped the lifeline of fashion? In fact, the designer of "Mashimaro" did not know it can be recognized when designing, so it can only say that consumers are the main body of manufacturing and pursuing fashion.

The Red Cross is an international aid and relief group developed during the war, and it has been popularized in 140 countries worldwide. In the Arab, it is marked by red crescent with white background; in Iran, it is marked by the red lion and sun with white background; and in the majority of countries, it is marked by red cross with white background. Although its name and logo may be different to a certain degree, the effect is the same. The reason for that is just the culture, religion and the historical origins are different in different places.

In summary, the graphic design works are actually a collection of thinking processes and symbolic expression. The symbolic elements it has selected, composed and used shall be of a certain special referent. In order to make symbol be able to play its role to the greatest degree, the high consistency with space, time and society shall be required. This has presented a very high requirement for designers, as they shall learn about various meanings that may possibly exist, that have existed ever before or the existing one to control all of its variables, in order to ensure that they can express their design ideas and inner feelings appropriately in the works. In fact, the design itself is a special symbol which is a medium to convey ideas. On the other hand, it is a combination of a lot of symbols through the selection, combination, transformation and regeneration by designers, to create a new symbol in line with their own thoughts, and is re-accredited by the public. Only when designers have achieved effective communication with the public, and have conveyed accurate information of their own, can they be said to have truly completed this thinking process. Symbol is a comprehensive interdisciplinary science, so research and practice in various disciplines cannot be inseparable from its profound principles and methodology. Of course, the graphic design is no exception.

\section{REFERENCES}

[1] Liu Yang. Discussion on Application of Optical Illusion Picture in Product Design [J] Packaging Engineering. 2012.04: 26-28

[2] Zheng Guoxi. Application of Graphics in Advertising Design [ $\mathrm{J}$ ] Packaging Engineering. 2012.10: 36-39

[3] Lu Yiwen. "Significant Point" in Graphics Design" [J] Packaging Engineering. 2012.10:46-51

[4] Liang Jialin. A View at Bionics Design of Architectural Modeling in terms of Landscape Style [J]. Big Stage, 2010(03):11-15

[5] Xiong Qinqin. Visual Communication Design Art [J]. Hundred Schools in Arts, 2008(03):12-14.

[6] Yin Nianping, Zhao Siyi. Appreciation on Chinese Ancient Architectural Tracery Art [J]. Architectural Knowledge, 2007(03): 103-104

[7] Cao Fang. Visual Communication Design Principle [M]. Jiangsu Fine Arts Publishing House, 2005(01):2-6

[8] Yang Jiong, Zhang Chaoping. Study on Application of National Decorative Arts in Interior Design [J]. Big Stage, 2012(05):37-39 